\title{
Les récepteurs $R X R$ sont des co-régulateurs des gènes répondant à l'acide rétinoïque, aux hormones thyroïdiennes et à la vitamine $D$
}

Il existe quatre types de récepteurs de l'acide rétinoïque : les récepteurs $\alpha, \beta$ et $\gamma$, dont le ligand est, sans conteste, l'acide rétinoïque ; les récepteurs $\mathrm{RXR}$ qui, tout en étant reliés, sur le plan de leur structure, aux précédents, semblent avoir des particularités quant à leur liaison à leur cible d'ADN et à la fixation du ligand $\left(\mathrm{m} / \mathrm{s} n^{\circ} 3\right.$, vol. 6, p. $307 ; n^{\circ} 8$, vol. 7, p. 863). Jusqu'alors, un récepteur $\mathrm{RXR} \alpha$ était connu. Des résultats récents suggéraient qu'il pouvait y avoir, physiologiquement, des ligands appartenant à la famille des rétinoïdes mais différents de l'acide tout-trans rétinoïque, et contrôler des gènes particuliers, par exemple celui codant pour la protéine de liaison du rétinol de type 2 (CRBP 2, cellular retinol binding protein 2).

L'équipe de M. G. Rosenfeld (San Diego et La Jolla, CA, USA) vient maintenant d'isoler un ADNc codant pour un second membre de cette sousfamille, $\operatorname{RXR} \beta$, et de lui assigner un rôle nouveau, celui de co-activateur coopérant avec les récepteurs de l'acide rétinoïque, des hormones thyroïdiennes et de la vitamine $\mathrm{D}$ pour augmenter leur liaison à leurs cibles spécifiques [1]. Le point de départ de cette recherche est l'observation faite, depuis quelques années, que les récepteurs de l'acide rétinoïque, des hormones thyroïdiennes et de la vitamine $\mathrm{D}$, avaient besoin d'une protéine auxiliaire pour se fixer avec une haute affinité à leur élément d'ADN spécifique [2-4]. Afin de cloner un ADN complémentaire codant pour de tels corégulateurs, l'équipe de Rosenfeld mit au point une méthode originale de criblage en deux étapes d'une banque d'expression d'ADNc. Premièrement, 11 clones codant pour une protéine reconnaissant l'élément de réponse à l'acide rétinoïque (RARE) furent isolés. Ceux correspondant à d'authentiques récepteurs de l'acide rétinoïque furent éliminés. Les autres furent criblés à l'aide d'une sonde protéique marquée au ${ }^{35} \mathrm{~S}$, à savoir le récepteur $\beta$ de l'acide rétinoïque lui-même $(\operatorname{RAR} \beta)$. $\mathrm{m} / \mathrm{s} n^{\circ} 3$, vol. 8 , mars 92
Un clone fut ainsi isolé, qui codait pour une protéine apte, tout à la fois, à reconnaître l'élément d'ADN RARE du gène $R A R \beta$ et à former un complexe protéine avec la protéine $\operatorname{RAR} \beta$. Sa séquence révéla qu'il était très similaire à la protéine $\mathrm{RXR} \alpha$ précédemment caractérisée. $\mathrm{RXR} \alpha$ et $\beta$ sont identiques à $95 \%$ au niveau de leur domaine de liaison à l'ADN et à $90 \%$ au niveau du domaine de liaison du ligand. Les protéines $\mathrm{RXR} \beta$ aussi bien que $\mathrm{RXR} \alpha$ sont capables de former des hétérodimères avec le récepteur de l'acide rétinoïque (RAR) ainsi qu'avec les récepteurs de la vitamine $\mathrm{D}$ et des hormones thyroïdiennes $[1,5,6]$. La liaison de ces hétérodimères aux éléments de réponse spécifiques est nettement supérieure à celle des homodimères, de même que la réponse transcriptionnelle ainsi induite.

Ce sont des résultats tout à fait concordants qu'ont également rapportés M. Leid et al., du laboratoire de P. Chambon (Strasbourg, France) [7]. Ces chercheurs ont purifié la protéine de cellules Hela capable d'augmenter la fixation du récepteur RAR $\gamma$ à l'élement RARE du gène $R A R \beta$ et l'ont partiellement séquencé. Ils ont alors observé que cette activité correspondait à un récepteur $\mathrm{RXR} \beta$ dont ils avaient cloné le gène chez la souris (ainsi que ceux de récepteurs $\mathrm{RXR} \alpha$ et $\gamma)$. L'équivalent humain de l'ADNc de $\mathrm{RXR} \beta$ a alors été cloné et utilisé pour mener des expériences très similaires à celles résumées cidessus. De plus, l'équipe de P. Chambon a fait l'intéressante observation qu'une semblable activité de co-régulateur de gènes contrôlés par des éléments de réponse aux tétinoïdes existait dans des cellules de drosophile. Or, un gène de cet insecte, ultraspiracle, code pour une protéine dont la structure est très voisine de celle de RXR [8] et qui pourrait jouer le même rôle que ce dernier, par exemple en coopérant avec le récep- teur de l'ecdysone $[9,10]$ ou avec le produit du gène $E 75$ induit par cette hormone de la mue et codant lui aussi pour un récepteur présomptif de la même famille [11]. La présence de ces co-régulateurs peut être fonctionncllement essentielle puisque certains récepteurs, notamment ceux de l'acide rétinoïque, sont normalement à très faible concentration et qu'ils pourraient ainsi nécessiter la liaison préalable à des co-activateurs pour activer leurs cibles. Le rôle inhibiteur de l'oncogène $v$-erb $A$, l'équivalent viral de l'un des gènes codant pour les récepteurs de l'acide rétinoïque, sur la réponse physiologique à ces hormones et à l'acide rétinoïque pourrait être dû, en partie, à la " titration " des coactivateurs $\mathrm{RXR}$, rendus ainsi indisponibles pour coopérer avec les réceptcurs RAR et c-ErbA. La même hypothèse peut être proposée pour expliquer l'influence de la protéine hybride PML-RAR $\alpha$, issue du réarrangement chromosomique $\mathrm{t}(15 ; 17)$ associé aux leucémies aiguës promyélocytaires, sur la différenciation granulocytaire $\left(\mathrm{m} / \mathrm{s} n^{\circ} 8\right.$, vol. 7, p. 880). La présence de plusieurs molécules de ce type, dont la diversité ne se limite peut-être pas à $\mathrm{RXR} \alpha, \beta, \gamma$, pourrait également permettre d'étendre l'éventail des actions biologiques dans lesquelles peuvent intervenir des récepteurs. Leur pouvoir activateur ou inhibiteur et la spécificité de leur liaison à certaines cibles peut, en effet, dépendre de la nature du corégulateur auquel ils sont liés. Demeure cependant au moins une ambiguïté importante quant au rôle physiologique des molécules RXR : celle de leur spécificité de ligand. Celle-ci, précédemment supposée $(\mathrm{m} / \mathrm{s}$ $n^{\circ} 8$, vol. 7, p. 863), a été confirmée par deux équipes importantes, celle des laboratoires Hoffman-La Roche (Nutley, NJ, USA) et celle de chercheurs californiens (San Diego et La Jolla) et texans (Houston) qui propo-

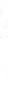

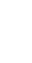

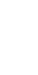

?

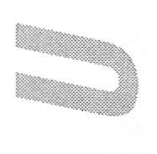


sent que le ligand physiologique des homodimères $\operatorname{RXR} \alpha$ soit l'acide 9-Cis rétinoïque, un stéréo-isomère de l'acide tout-trans rétinoïque $[12,13]$. L'acide 9-Cis rétinoïque, produit d'isomérisation in vivo de l'acide rétinoïque, est un aussi bon activateur des récepteurs RAR que l'acide tout-trans rétinoïque et est 40 fois plus actif que ce dernier sur les récepteurs RXR [13]. Des expériences complémentaires diront si la formation des hétérodimères est capable, non seulement de changer la spécificité de liaison à l'ADN et le pouvoir activateur ou inhibiteur sur la transcription, mais aussi l'affinité pour différents ligands et, par conséquent, leurs effets. On peut aussi imaginer que les deux ligands d'un hétérodimère composé de RXR et d'un autre récepteur soient susceptibles de contrôler finement la réponse à l'un et à l'autre, voire de créer une combinatoire de signaux engendrant des " messages " particuliers.

A. $\mathbf{K}$.
1. Yu VC, Delsert $C$, Andersen $B$, et al. RXR $\beta$ a co-regulator that enhances binding of retinoic acid, thyroid hormone and vitamin $\mathrm{D}$ receptors to their cognate response elements. Cell 1991; 67 1251-66.

2. Burnside J, Darling DS, Chin WW. A nuclear factor that enhances binding of thyroid hormone receptors to thyroid hormone response element. $J$ Biol Chem 1990 ; 265 : 2500-4.

3. Glass CK, Devary OV, Rosenfeld MG. Multiple cell type-specific proteins differentially regulate target sequence recognition by the $\alpha$ retinoic acid receptor. Cell 1990 ; 63 : 729-38.

4. Liao J, Ozono K, Sone P, McDonnel DP, Pike JW. Vitamin D receptor interaction with specific DNA requires nuclear protein and 1,25-dihydroxy vitamin D3. Proc Nall Acad USA 1990 ; 87 : 9781-8.

5. Kliewer SA, Umesono K, Mangelsdorf DJ, Evans RM. Retinoic X receptor interacts with nuclear receptors in retinoic acid, thyroid hormone and vitamin $\mathrm{D}_{3}$ signalling. Nature $1992 ; 355$ : 446-9.

6. Zhang XK, Hoffmann B, Tran PBV, Graupner G, Pfahl M. Retinoic X receptor is an auxiliary protein for thyroid hormone and retinoic acid receptors. Nature 1992 ; 355 : 441-6.

7. Leid M, Kastner P, Lyons R, et al. Purification, cloning, and RXR identity of the Hela cell factor with which RAR or TR heterodime- rizes to bind target sequences efficiently. Cell $1992 ; 68$ : 377-95.

8. Oro AE, McKeown M, Evans R M. Relationship between the product of the Drosophila ultraspiracle locus and the vertebrate retinoid $\mathrm{X}$ receptor. Nature 1990 ; 347 : 298-301.

9. Koelle MR, Talbot WS, Segraves WA, Bender MT, Cherbas P, Hogness DS. The Drosophila $E c R$ gene encodes an ecdysone receptor, a new member of the steroid receptor superfamily. Cell $1991 ; 67$ : 59-77.

10. Segraves WA, Hogness DS. The E75 ecdysone-inducible gene responsible for the 75B early puff in Drodophila encodes two new members of the steroid receptor superfamily. Genes Dev 1990 ; 4 : 204-19.

11. Segraves WA. Something old, some things new : the steroid receptor superfamily in Drosophila. Cell $1991 ; 67$ : 225-8.

12. Levin AA, Sturzenbeckor LJ, Kazmor S et al. 9-Cis retinoic acid stereoisomer binds and activates the nuclear receptor $\operatorname{RXR} \alpha$. Nature 1992 ; 355 : 359-61.

13. Heyman RA, Mangelsdorf DJ, Dyck JA, et al. 9-Cis retinoic acid is a high affinity ligand for the retinoid X receptor. Cell $1992 ; 68$ : 397-406.
Une protéine du stress se comporte comme un thermomètre moléculaire. Le mécanisme par lequel les bactéries sont capables de sentir la température extérieure et, dans certaines limites, de s'y adapter, notamment via l'induction des gènes codant pour les protéines du stress, restait jusqu'à il y a peu complètement inconnu. L'équipe de McCarthy et Walker, du MIT (Cambridge, MA, USA), vient de détecter un premier thermomètre moléculaire qui est lui-même une protéine du stress, la protéine HSP 70 (heat shock protein) d'Escherichia coli, codée par le gène DnaK. Il était déjà connu que HSP 70, une protéine chaperon $\left(\mathrm{m} / \mathrm{s} n^{\circ} 5\right.$, vol. 7, p. 496), se complexe à d'autres protéines qu'elle aide à acquérir une conformation correcte. De ce fait, les heat shock prolains peuvent se lier à des molécules polypeptidiques partiellement dénaturées au cours du choc thermique et leur permettre de recouvrer une conformation, c'est-à-dire une activité biologique normale. Cependant, la libération par HSP 70 d'une protéine à laquelle elle est liée nécessite l'hydrolyse d'un ATP. HSP 70 existe sous deux formes, l'une phosphorylée et l'autre déphosphorylée, seule la première étant capable d'hydrolyser l'ATP, et par conséquent de libérer sa proie protéique. Le degré de phosphorylation des protéines HSP 70 semble être progressif au est donc une mesure directe. Parmi les protéines se liant à HSP 70 chez $E$. coli, le facteur $\sigma 32$, protéine auxiliaire de l'ARN polymérase II, semble jouer un rôle particulier. $\mathrm{Ce}$ facteur $\sigma$ agit, en effet, pour stimuler la transcription des gènes codant pour les protéines du stress. Par conséquent, HSP 70 " sent " l'augmentation de la température en augmentant son degré de phosphorylation, ce qui entraîne une dissociation d'avec les protéines liées, lui permettant d'assurer sa fonction de chaperon vis-à-vis d'autres molécules risquant d'être dénaturées par la chaleur. Parmi les protéines libérées se trouve le facteur $\sigma 32$ qui accroît encore l'efficacité du système en stimulant la transcription des gènes des protéines du choc thermique, notamment de celui de HSP 70. Les fonctions d'autophosphorylation et d'activité ATPasiques de HSP 70 sont autonomes, c'est-à-dire sont liées à la molécule ellemême, qui joue, de œe fait, le rôle d'un véritable thermomètre endocellulaire.

[1. McCarthy JS, Walker GC. Proc Natl Acad Sci USA 1991 ; 88 : 9513-7.]

Des jumeaux de pères différents. L'ovulation chez la femme a, en général, comme résultat un ovule unique. Lorsqu'elle en produit plus d'un peuvent naître des jumeaux dizygotes, et il peut arriver que chacun d'eux soit fécondé par un homme différent. Les exemples connus restent rares. Le plus fameux est celui d'une femme qui, en 1810, accoucha d'un enfant blanc et d'un mulâtre. En 1940, un cas fut reconnu par typage des antigènes des globules rouges, confirmé, près de 40 ans plus tard, par un typage HLA [1, 2]. Verma et al. (Brooklyn, NY, USA) rapportent [3] un exemple de jumeaux dizygotes (un garçon et une fille), dans lequel le père allégué renvoya la responsabilité au père légal (qui, pour sa part, refusa toute recherche de paternité), mais accepta un exarnen cytogénétique. La présence à l'état homozygote chez le père allégué, d'un marqueur peu fréquent sur le chromosome 18 permit d'éliminer sa paternité chez la fille qui ne le possédait pas. Au contraire, le garçon portait ce marqueur sur un de ses chromosomes 18. Ce résultat, joint à d'autres caractères comme la taille du chromosome $Y$, plaidait en faveur de la responsabilité du père allégué. Averti de ces résultats, le père devenu probable refusa la confirmation par empreintes génétiques. On sait encore que la mère admit la participation possible des deux hommes, mais on ignore si l'un d'eux s'occupe des enfants.. [1. Geyer E. in [3].]

[2. Terasaki PL, et al. N Engl J Med 1978 ; 299 : 590-2.]

[3. Varma RS, et al. Loncet $1992 ; 339$. 63-4.] 